\title{
Advancing Elite Athlete Mental Health Treatment with Psychedelic-Assisted
}

\section{Psychotherapy}

\author{
Courtney C. Walton ${ }^{1,2, *}$ Paul Liknaitzky ${ }^{3}$
}

Please check for any bibliographic updates and cite the published version of this manuscript:

Walton, C. C., \& Liknaitzky, P. (2020). Advancing Elite Athlete Mental Health Treatment with Psychedelic-Assisted Psychotherapy. Journal of Applied Sport Psychology. doi:10.1080/10413200.2020.1848941

\author{
Affiliations \\ ${ }^{1}$ Orygen, Melbourne, Australia. \\ ${ }^{2}$ The University of Melbourne, Melbourne, Australia. \\ ${ }^{3}$ School of Psychology, Deakin University, Geelong, Australia \\ C. Walton ORCID: 0000-0003-0835-2310 \\ P. Liknaitzky ORCID: 0000-0001-5690-2263
}

\section{* Correspondence should be addressed to Dr Courtney C. Walton.}

E-mail: courtney.walton@ @rygen.org.au Address: Orygen, 35 Poplar Road, Parkville 3052, Melbourne Victoria, Australia

Running Heading: Psychedelics in elite sport

Word Count: 5,624

Funding: No sources of funding were used to assist in the preparation of this article

Conflicts of interest/Competing interests: C Walton: None. P Liknaitzky: In accordance with Taylor \& Francis policy and my ethical obligation as a researcher, I am reporting that I am a scientific advisor to a company that may be affected by the research reported in the enclosed paper. I have disclosed those interests fully to Taylor \& Francis, and I have in place an approved plan for managing any potential conflicts arising from that involvement.

Acknowledgements: The ideas contained within this manuscript are those of the authors, and do not necessarily reflect those of Orygen, the University of Melbourne, or Deakin University. We would like to thank A/Prof Rosie Purcell, A/Prof Simon Rice, Dr Ruben Laukkonen, and Dr Claire O'Callaghan for providing critical feedback during the preparation of an earlier version of this manuscript. 


\begin{abstract}
Despite a politically vilified past, classical psychedelics, including lysergic acid diethylamide (LSD), psilocybin, and dimethyltryptamine (DMT), are experiencing a revival in scientific and clinical research. When used under the appropriate guidance and setting, these substances show promise for substantially improving well-being and reducing mental ill-health alongside an excellent safety profile. Elite athletes are known to experience mental health disorder symptomatology and psychological distress at similar, if not higher, rates to the general population. Therefore, this promising line of research may be relevant to mental health treatment within elite sport. Psychedelic treatment may reduce the incidence and experience of mental ill-health in athletes, particularly when related to a range of issues commonly seen in elite sport, including therapeutic resistance, challenges to identity and meaning through career transitions and injury, and managing interpersonal stress and conflict. As a number of psychedelic treatments are currently advancing through the drug development pathway in the US and EU, it is timely to develop an understanding of the clinical application of psychedelics within elite sport, and the legislative and sport-specific regulations that will need to be addressed if psychedelics become registered medicines. In this article, we outline the sport-specific relevance of psychedelic treatments, the role of sports psychologists and psychiatrists in delivering and managing prospective psychedelic treatment, the key ethical and regulatory issues this prospective treatment raises, as well as propose initial research questions the field could address. We argue that psychedelic-assisted psychotherapy should be investigated as a novel treatment option for addressing mental ill-health in elite athletes.
\end{abstract}

Key Words: Psychedelics, hallucinogens, psilocybin, LSD, mental health, elite athletes, sport 


\section{Lay Summary:}

Psychedelic-assisted treatments are attracting increased attention and impressive efficacy in early psychiatry research. We outline the potential for psychedelic therapies to treat the mental health problems seen in athletes. We describe the rationale for their use specifically within elite athletes, the sport-specific considerations and challenges that need to be addressed, the role of sports clinicians, and define the research required to demonstrate their feasibility, safety and efficacy within athletes.

\section{Implications for Practice:}

- With poor mental health a growing concern in elite sport, the clinically-supported application of psychedelics may be well positioned to treat these issues and the sportspecific factors that can contribute to them.

- Various regulatory and sport-specific ethical factors will make research in this context challenging; however, these are tractable, and preliminary research in the application of psychedelic treatments for mental ill-health associated with elite sport is warranted.

- There is no evidence yet to support the safety and efficacy of psychedelic treatment in elite sport, and psychedelic compounds are not currently registered for medical use. Accordingly, the ideas explored within this commentary should not be taken as support for independent recreational use of psychedelics. 


\section{Advancing Elite Athlete Mental Health Treatment with Psychedelic-Assisted}

\section{Psychotherapy}

In recent years there has been a dramatic increase in both research and clinical attention to the prevalence of mental ill-health in elite sport (Reardon et al, 2019). Current evidence indicates similar rates of mental health problems in athletes compared with the general population (Gouttebarge et al., 2019; Reardon et al., 2019; Rice et al., 2016). Among other stressors (Arnold \& Fletcher, 2012), pressure to perform, overtraining, poor performance, interpersonal conflict, injury, and retirement at a young age may all contribute to psychological distress (Poucher, Tamminen, Kerr, \& Cairney, 2019). As increasing rates of mental ill-health are uncovered among elite athletes, researchers and practitioners now scramble to assemble sport-specific and contextually appropriate assessment and treatment for athletes (Purcell, Gwyther, \& Rice, 2019).

Given the significant need for better mental health treatment of athletes and the likelihood that psychedelics will become approved medications in the near-future, in this paper we discuss the highly promising results of psychedelic-assisted psychotherapy for high-prevalence conditions commonly seen in elite sport. In addition, there is substantial public and clinical interest in psychedelic medicine, with the potential for widespread acceptance and application should they be approved. Given this, here we outline key alignments between outcomes associated with psychedelic treatments and sport-specific factors that contribute to mental ill-health. As psychedelic-assisted psychotherapies are poised to be among the most significant advances in modern psychiatry, the potential application of this treatment approach in elite athletes should be explored. We suggest that practitioners in sport psychology and psychiatry would do well to be appraised of the field in anticipation of an upsurge in interest from their clients. Psychedelic-assisted therapies entail both pharmacological and psychotherapeutic elements of treatment, and consequently a 
collaborative care model between sport psychiatrists, psychologist and other clinicians is likely to be the most feasible and potentially beneficial approach. Moreover, sport psychology practitioners who do not directly deliver the treatment may still be well placed to coordinate and facilitate such treatment for athletes. To our knowledge, no research to date has proposed or assessed the application of psychedelics in elite sport, and thus this paper represents an initial exploration of the rationale and considerations.

\section{Clinical Psychedelic Research}

In order to understand the current state of psychedelic research and how it may be applied to elite sport, one must briefly step back and revisit the past. The term psychedelic was proposed by psychiatrist Humphry Osmond (Osmond, 1957), and stems from the Greek for "mind-manifesting". The term is generally used to refer to a group of tryptamines that act on the serotonergic system, and produce most of their subjective effects through agonism of the 5-HT2A receptor (Nichols, 2016). Examples include lysergic acid diethylamide (LSD), psilocybin (found within certain genera of mushroom), and dimethyltryptamine ${ }^{1}$. Many of the plant-based psychedelics are among the oldest psychopharmacological agents known to be used by humans, with traditional use in the context of healing, knowledge acquisition, and ceremony dating back millennia (Hofmann \& Ott, 1980; Nichols, 2016; Schultes, 1969).

In the 1950s, psychiatric research began to suggest that the supported administration of psychedelics may be able to treat mental illness, with impressive results across a range of conditions (Grinspoon \& Bakalar, 1981). Whilst the scientific rigour of many studies from this period were poor by modern standards, by the late 1960s psychedelic medicine was rapidly becoming a leading school of psychiatry, practiced or supported by prominent psychiatrists at the time (Grinspoon \& Bakalar, 1979). Over 40,000 mental health patients

\footnotetext{
${ }^{1}$ While medicinal MDMA is often loosely referred to as a psychedelic, this paper only addresses the utility of the classical psychedelics listed.
} 
were administered LSD, and over 1,000 peer-reviewed articles published (Grinspoon \& Bakalar, 1981). The sudden drop in research occurred not due to a lack of scientific or medical progress, but rather the politicisation of psychedelics, and the orchestration of drug scheduling, funding cuts, and cultural stigma that served to suppress the anti-war and antiestablishment views associated with people who used psychedelics (Nichols, 2016).

Over the last two decades, a growing resurgence in clinical psychedelic research has gained considerable momentum, with ground-breaking studies demonstrating the safety and therapeutic potential of psychedelic-assisted psychotherapy. In most studies, the substance is given once or twice under supervision alongside a brief course of psychotherapy that allows participants to work with their specially trained therapists to prepare for, safely undergo, and subsequently integrate the psychedelic experience (Nutt, Erritzoe, \& Carhart-Harris, 2020). During the participants' 'trip', therapists most commonly take a patient-led and supportive approach. Studies using this method have examined the role of psychedelics in both health and disease, and have begun to unravel the neurophysiological processes underlying their effects and the workings of the brain more broadly (Carhart-Harris et al., 2014; CarhartHarris, 2018; Vollenweider and Preller, 2020).

While much of the current work should be considered as preliminary, results are extremely promising. Large reductions in recurrent depression have been shown using one low and one full psilocybin dose in an open-label pilot trial (Carhart-Harris et al., 2016) with over half of the mean clinical effect sustained at six months (Carhart-Harris et al., 2018). A number of trials have shown a reduction in anxiety relating to life-threatening medical conditions using both LSD and psilocybin (Gasser et al., 2014; Griffiths et al., 2016; Grob et al., 2011; Ross et al., 2016). In small pilot trials, psilocybin has been shown to be efficacious in treating obsessive-compulsive disorder (Moreno, Wiegand, Taitano, \& Delgado, 2006), smoking addiction (Johnson, Garcia-Romeu, Cosimano, \& Griffiths, 2014) and alcohol 
dependence (Bogenschutz et al., 2015). There are currently a large number of ongoing projects seeking to replicate and extend clinical psilocybin research within Phase II trials (see Meikle et al., 2019).

The benefits of psychedelics on mental health extend beyond psychiatric disorders, with growing evidence in the field of positive psychology (Jungaberle et al., 2018; Keyes, 2002; Uphill, Sly, \& Swain, 2016). Across numerous studies, experimentally administered or naturalistic use of psychedelics were linked to acute and long-term improvements in mood, prosocial behaviour and empathy, cognitive flexibility and creativity, openness, and mindfulness-related capabilities, among other changes (Jungaberle et al., 2018). For example, in a counterbalanced double-blind design (Griffiths, Richards, McCann, \& Jesse, 2006), participants administered with psilocybin compared with active placebo reported increases in positive affect, and a range of personally profound and meaningful experiences which were corroborated by family, friends, and colleagues. At 14-months follow-up, 58\% of participants rated the experience as being in the five most personally meaningful experiences of their lives. Relatedly, following a single psilocybin session, significant and lasting increases in the personality trait 'openness' were found; the first time a sustained change within typically stable personality traits has been experimentally produced (MacLean, Johnson, \& Griffiths, 2011). These, along with a growing number of studies, highlight the potential for well-supported psychedelic experiences to have a profound and long-lasting positive impact on an individual (Aday, Mitzkovitz, Bloesch, Davoli, \& Davis, 2020). Notwithstanding some impressive results in terms of the size, speed, and the sustained nature of beneficial changes associated with psychedelics, various methodological limitations warrant caution. For example, many studies in the modern era of psychedelic research have been small (albeit with large enough effect sizes to be adequately powered in 
many cases), and hampered by difficulties with adequate blinding, outsized expectancy effects, and participant self-selection biases (Meikle et al., 2019; Reiff et al., 2020).

\section{Potential Risks of Use}

Further to demonstrated clinical safety and efficacy, increasing acceptance of psychedelics as medicines has come from additional evidence showing that the risk of harm caused by psychedelic use is low even in 'the wild', particularly in comparison to other legal or illicit drugs (Bonomo et al., 2019; Nutt, King, \& Phillips, 2010; van Amsterdam, Nutt, Phillips, \& van den Brink, 2015). Indeed, a strong case has been made for the downscheduling of psychedelics in the United States from Schedule 1 (no currently accepted medical use and a high potential for abuse) to Schedule 4 (low potential for abuse and low risk of dependence) (Johnson, Griffiths, Hendricks, \& Henningfield, 2018). Classical psychedelics do not produce dependence, withdrawal, toxic effects to the brain and other organs, or addictive behaviours (Johnson, Richards, \& Griffiths, 2008; Johnson et al., 2018; Nichols, 2016). At a population level, the use of psychedelics is associated with reduced, rather than increased, mental health problems (Johansen \& Krebs, 2015; Krebs \& Johansen, 2013).

However, notable risks are associated with classical psychedelics. Contrary to common public perception, the link to triggering psychosis or psychotic behaviour is unclear and extremely rare, more likely representing prior vulnerabilities than direct drug effects (Johansen \& Krebs, 2015; Krebs \& Johansen, 2013). The key risks of psychedelics are acute distressing psychological experiences, characterized by anxiety, panic, dysphoria, and/or paranoia (Johnson et al., 2008). At their extreme, they can result in trauma and lasting negative psychological effects (Carbonaro et al., 2016). While such risks should not be taken lightly, they are almost entirely mitigated in the modern clinical context through adequate screening and expert support from appropriately trained clinicians. Indeed, surveyed 
researchers working with LSD or mescaline in the 1950s reported no lasting adverse side effects across roughly five thousand participants (Cohen, 1960), and no serious adverse events or long-term adverse effects have been reported across over 2,000 psilocybin dosing sessions since the 1990s (Ross et al., 2016). With skilful supervision and significant consideration for participant preparation and the physical and interpersonal setting, challenging experiences under psychedelics are frequently reported to be highly beneficial and therapeutically efficient (Carbonaro et al., 2016; Carhart-Harris et al., 2018; Fadiman, 2011; Johnson et al., 2008).

\section{Psychedelic Targets in Sport}

There is initial evidence that psychedelic-assisted psychotherapy appears to be capable of providing safe, long-lasting, and meaningful changes under certain conditions. The effects have been replicated in a number of populations and across indications, suggesting that therapeutic mechanisms may be in part transdiagnostic. Given the field's relatively early stage of development, this already wide set of applications indicates that psychedelics may soon prove beneficial for further specific populations. While there is currently no experimental evidence of psychedelic use in sport, features of mental ill-health among athletes may lend themselves particularly well to this treatment approach: (1) resistance to help-seeking, and to challenging feelings, are high among elite athletes; (2) mental health disorders are common in elite athletes, with some studies indicating higher rates of mental ill-health than non-specific community samples; (3) interpersonal conflict is common in elite sport, and occurs alongside a substantial need to cooperate and perform as part of a team; and (4) retirement from elite sport, typically at a younger age than in most professions, is commonly accompanied by a major loss of identity and meaning. We emphasise that this proposal does not argue that athletes are in some way more suited to psychedelic medicine than other populations. Rather, we argue that if sport-specific 
considerations around doping and performance can be investigated and resolved, athletes may be no less suited to testing this treatment option than perhaps intuitively assumed. Moreover, psychedelic treatment appears to afford changes directly relevant to key features of mental ill-health in athletes. In this section, we outline the potential alignment of psychedelic treatment within elite sport. This list is by no means exhaustive, but seeks to illustrate a number of plausible uses for psychedelic medicine in this context.

\section{Treatment of Common Mental Health Disorders}

While there are sport-specific factors that can precipitate poor mental health, highprevalence mental health disorders occur commonly in elite athletes. A recent study suggested higher rates of psychological distress and probable mental health disorder in Australian elite athletes than in the general population (Purcell, Rice, Butterworth, \& Clements, 2020), while another showed $52 \%$ of Swedish elite athletes had experienced mental health problems at some point with a $12 \%$ point prevalence (Åkesdotter, Kenttä, Eloranta, \& Franck, 2020). More broadly, meta-analysis suggests that $34 \%$ of elite athletes report symptoms of anxiety and/or depression and 19\% report symptoms of alcohol misuse (Gouttebarge et al., 2019). Such figures show that athletes are far from immune to common psychological disorders. As documented above, psychedelic-assisted psychotherapy has been shown to be efficacious for depression, anxiety, and harmful alcohol use (Rucker, Iliff, \& Nutt, 2018), with larger studies in progress (Nutt et al., 2020). Therefore, if support for the safety and efficacy of psychedelics continues to grow, while treatment options in elite athletes remain limited, it is reasonable to consider the merits of psychedelic medicine as a novel therapeutic option.

Psychedelic treatments may also circumvent issues specific to long-term psychiatric medication, including difficulties maintaining peak athletic performance, and managing doping regulations (Chang et al., 2020). Treatment programs in the modern era typically last 
only seven to twelve weeks; they include one or two all-day dosing sessions, with about three 'preparatory' psychotherapy sessions prior to the first dose, and about three ‘integration’ psychotherapy sessions following each dose. Such treatment protocols have been shown to produce clinical benefits that endure for many months, and in some cases years, for a large proportion of patients. Psychedelic medicine may therefore offer a less disruptive intervention for athletes, and may be better suited to the scheduling barriers and difficulty with retention observed in elite athletes engaging in therapy (Stillman et al., 2019). Moreover, as these treatments are relatively brief, and the biological half-life of psychedelics is only a few hours, potential future complications associated with doping may be reduced or eliminated if athletes are not competing during the short treatment program.

\section{Reducing Resistance to Treatment}

There are significant barriers to athletes engaging in mental health treatment (Chang et al., 2020). The ideal of 'mental toughness' is common, and there is evidence to suggest that athletes often do not publicly report mental health concerns or seek psychological assistance (Gulliver, Griffiths, \& Christensen, 2012; Moreland, Coxe, \& Yang, 2018; Watson, 2005). For this reason, programs targeting mental health in athletes have proposed terminology contextualised within performance and optimization, rather than speaking directly to mental health disorders (Donohue et al., 2018; Poucher et al., 2019). Younger male athletes in particular appear to hold negative attitudes towards help-seeking; a response potentially related to a range of cultural and societal pressures to do with masculinity (Doherty, Hannigan, \& Campbell, 2016; Rice, Purcell, \& McGorry, 2018).

Psychological resistance to challenging feelings and to help-seeking, common in elite athletes, may be effectively addressed by psychedelics' ability to increase trait openness (Griffiths et al., 2006), increase acceptance (Luoma, Sabucedo, Eriksson, Gates, \& Pilecki, 2019), and potentially lower psychological defences (Carhart-Harris et al., 2012; Cohen, 
1967). Moreover, psychedelics commonly and substantially increase self-worth and selfcompassion (Watts, Day, Krzanowski, Nutt, \& Carhart-Harris, 2017) - factors associated with psychological distress in athletes (Walton, Baranoff, Gilbert, \& Kirby, 2020) affording greater acknowledgement of one's maladaptive thoughts, feelings and behaviours, and the imperative to address these. Given psychedelics have been employed towards positive psychology outcomes (Jungaberle et al., 2018), the ability to frame psychedelic treatments in this way may increase acceptability within sport.

\section{Improving Relationships}

The world of elite sport is one in which interpersonal conflict is common (Wachsmuth, Jowett, \& Harwood, 2017). Elite sport requires a range of highly ambitious individuals, with differing perspectives and personalities, to spend large amounts of time working closely together under extreme pressure and fatigue. The potential for conflict to occur is high: between an athlete and their teammates, coaches, management, media, or fans. Increasing the ability for athletes to cope with interpersonal conflict will have important consequences for both well-being and performance (Wachsmuth et al., 2017).

To this end, psychedelics have been shown to increase interpersonal perceptiveness (Griffiths et al., 2006), empathy (Schmid et al., 2015), and prosocial behaviour (Dolder, Schmid, Müller, Borgwardt, \& Liechti, 2016; Griffiths et al., 2018; Preller et al., 2015). For example, Belser and colleagues found that positive changes to perceptions of previously difficult relationships was one of the core themes reported following psilocybin treatment (Belser et al., 2017). Forgiveness and increased empathy were key aspects of this transformation, as participants were able to let go of long-standing negative feelings towards specific individuals. Psychedelics may afford the capacity for more constructive relational dynamics, thereby enhancing the ability to navigate conflict well, and improving team cohesion, athlete wellbeing, and performance. 


\section{Understanding Identity and Finding Meaning in Retirement and Injury}

Athletic identity foreclosure, an excessive and singular commitment to the athlete role and identity, is a key concern in sport. There is considerable evidence that it is associated with more difficulty in retirement, including longer adjustment periods, a greater loss of identity, and reduced well-being (Brewer \& Petitpas, 2017; Park, Lavallee, \& Tod, 2013; Ronkainen, Kavoura, \& Ryba, 2016). Similarly, stronger athletic identity is associated with increased depression during the injury rehabilitation process (Baranoff, Hanrahan, \& Connor, 2015; Brewer, Cornelius, Stephan, \& Van Raalte, 2010). These transition experiences can resemble a process of grieving, where an athlete must come to terms with the temporary or permanent loss of something personally essential - their athletic identity. A central source of meaning may need to be replaced if a retired athlete is to maintain their well-being. Retired athletes represent a key sample which may stand as a first group to investigate, given their established need for mental health treatment (Mannes et al., 2018), complex presentation around identity and transitions (Park et al., 2013), and crucially, their exclusion from the complex sporting regulatory considerations described in the following section.

Of relevance, psychedelics have been shown to engender a deeper understanding and acceptance for one's identity (Belser et al., 2017; Cavnar, 2014; Shanon, 2010), produce lasting changes in personality traits (Griffiths et al., 2006), and substantially increase selfcompassion (Sampedro et al., 2017). It is therefore conceivable that psychedelics may assist in the process of acceptance and understanding of 'athlete identity' loss, and a renewed and more holistic sense of self. Anecdotally many athletes have spoken about the feeling of 'dying twice', so while clearly distinct from this loss, there are nevertheless some thematic similarities to those experienced by patients with life-threatening illness, including the process of coming to terms with a major obstacle outside of one's control, and a loss of 
previously conceived meaning and purpose that can lead to hopelessness (Rosenbaum et al., 2019). Across multiple trials, terminally-ill patients who received psilocybin have demonstrated reductions in existential distress, improved quality of life, and an increased capacity to accept difficult change and loss (Ross, 2018). In addition, psychedelics produce both dramatic acute increases in the sense of experiential meaningfulness (Garcia-Romeu et al., 2019; Preller et al., 2017), and longer term meaning and purpose (Griffiths et al., 2016; Griffiths et al., 2006; Watts et al., 2017). These findings indicate that psychedelic-assisted psychotherapy may have something to offer retired and severely injured athletes experiencing related psychological distress, through an enhanced ability to come to terms with loss and identity change, and through potentially finding meaning and purpose beyond sport.

\section{Sport-Specific Considerations around Substance Use}

The introduction of psychedelic research within the population of elite athletes would require careful consideration of sport-specific factors. Despite some ongoing conservative views around psychedelic medicine (Puspanathan, 2017; Strauss, Bright, \& Williams, 2016), there is no legal impediment to conducting clinical trials with these scheduled substances, and numerous research groups within highly esteemed institutions are currently conducting high quality clinical trials. The tide is clearly turning in clinical psychedelic research globally, and Australia has now joined the field with a number of psychedelic trials that are either active or approved (co-author PL is an investigator on a number of these trials). However, with respect to the application of psychedelic medicine within elite sport, a range of sport-specific considerations around performance and regulation must be considered, which will make investigation of their efficacy more complex than in the general population. Here, we touch on their potential interactions with performance, and current scheduling by the World Anti-Doping Agency (WADA). 


\section{Possible Effects on Performance}

While this paper outlines the potential utility of psychedelic medicine for treating mental ill-health in elite athletes, the potential for psychedelics to also impact athletic performance - in either positive or negative ways - must be addressed. While there are both anecdotal (Oroc, 2011) and experimental (Polito \& Stevenson, 2019) reports that indicate the potential for low doses of psychedelics to enhance some forms of performance (e.g., through flow-like states, increase stamina and balance, increased attentional focus), these reports relate to acute (not enduring) effects. Given psychedelic treatment entails a full dosing day under clinical supervision, athletes would not be competing while under the influence of psychedelic dosing. Although evidence for relevant performance enhancement is scant, and restricted to the dosing day, future studies should directly test a number of possible ways in which athletic performance might be enhanced beyond improved mental health and beyond the acute effects of the psychedelic.

In addition to potential performance enhancement, we must consider any possible detrimental effects to performance. Such plausible risks include the transient occurrence of unresolved challenging emotional experiences associated with treatment, and very rare postacute perceptual distortions (e.g., hallucinogen persisting perception disorder; Lerner et al., 2002), or other shorter-term distortions to visual or spatial processing) that could interfere with athletic performance (Johnson et al., 2008). While the risk of serious or prolonged adverse effects is extremely low when participants are adequately screened (for example, for psychosis risk) and have appropriate clinical support and context (Johnson et al., 2008), their possible impact on sporting performances should be considered and mitigated.

\section{Psychedelics and Doping Regulations}

Perhaps even more so than in the general population (Sellers and Leiderman, 2018), conducting research with these substances will be complex, given the many additional 
regulations that athletes must adhere to. Whether psychedelic medicine might be considered a form of doping in sport will need to be determined by regulatory bodies like WADA. As highlighted by Mazzoni and colleagues (2017), classical psychedelics are unique among most other illicit drugs in that they are not currently listed by WADA among "Prohibited Substances" 2 (WADA, 2020). However, WADA also make reference to "Non-Approved Substances", which include those which are unlisted but have "no current approval by any governmental regulatory health authority for human therapeutic use (e.g. drugs under preclinical or clinical development)" (WADA, 2020). We envisage that Therapeutic Use Exemptions would be required for currently active athletes to engage in any clinical trial investigating psychedelic-assisted psychotherapy.

If psychedelics become approved medicines in the general public, WADA may need to specifically classify these substances and methods. WADA outlines that it will list a substance on the prohibited list where two of the following three criteria are met: (1) "Medical or other scientific evidence, pharmacological effect or experience that the substance ... has the potential to enhance or enhances sport performance"; (2) "Use of the substance or method represents an actual or potential health risk to the athlete"; and (3) "Use of the substance or method violates the spirit of sport described in the introduction to the Code" (WADA, 2020). Currently there is no evidence to suggest classical psychedelics meet two of these three criteria (Mazzoni et al., 2017). Undoubtedly this could change if future research found that there are any potential post-acute dosing effects of psychedelics on performance as relevant to Criterion 1, though as described elsewhere in this manuscript this would appear unlikely. Criterion 2 is not justifiable given the excellent safety profile demonstrated for psychedelics when used in clinical settings. Finally, Criterion 3 relies on a

\footnotetext{
${ }^{2}$ See https://www.wada-ama.org/sites/default/files/wada 2020 english prohibited list 0.pdf for a full and current list with details regarding 'Non-Approved' and 'Prohibited' Substances.
} 
range of moral viewpoints and biases which are difficult to accurately argue (for or against) in the current context of clinical psychedelic use. We do not, however, see a clear rationale for why their use in treating mental ill-health would violate the spirit of sport, particularly if performance enhancement is not evidenced.

The use of psychiatric medication in sport is already complex, with a range of known but unwanted performance enhancing and detrimental side effects possible (see Reardon, 2016). Current evidence indicates that clinically-supported psychedelic treatments pose a minimal risk that falls below that of the second criteria above (risk of harm to the athlete). If future empirical studies show that psychedelic-assisted psychotherapy enhances performance beyond improved mental health, then WADA's criteria 1 and 3 may become relevant, and this would require complex ethical decision making. We anticipate that treatment would still be possible if the benefit to an athlete's mental health is deemed sufficient enough to override doping concerns, again through the use of Therapeutic Use Exemptions. To begin ascertaining whether psychedelic medicine could be impacted by doping concerns in elite athletes, future trials must test key variables relevant to athletic performance during, and in the days subsequent to, psychedelic dosing.

\section{Avenues for Future Research}

As outlined above, future research could potentially employ psychedelic-assisted psychotherapy to address common mental health disorders in athletes, including resistance to mental health help-seeking, common and high-stakes interpersonal conflict, and loss of meaning or identity crises associated with protracted injury or retirement that often occur at a young age.

To explore the application of psychedelic medicine within elite sport, various barriers and limitations require further research and consideration. While there is a growing evidence base for the safe and efficacious use of clinical psychedelics to treat mental ill-health, the 
body of contemporary research (as opposed to pre 1970s) is still relatively modest. In addition, while the safety profile of modern clinical psychedelic trials has been excellent, real risks are associated with their application, including acute psychological distress and the potential for traumatic experiences. Of note, individuals with a risk for psychosis, Borderline Personality Disorder, and Bipolar Disorder are typically excluded from research trials for safety concerns. Also, as this treatment approach is markedly distinct from anything else in the clinical armamentarium, qualified mental health clinicians with specialist psychedelic training should deliver treatment within research trials (Johnson et al., 2008). Future studies should be designed to directly investigate whether classical psychedelics administered to healthy athletes significantly improve (post-acute) performance across a range of sporting activities. Moreover, the sport-specific issues of professional contract restrictions and marketability relating to image rights or sponsorship, in addition to the risk of conflating treatment with doping, will need to be addressed. However, given the potential for benefit to professional athletes suffering with mental illness, particularly in severe cases (Baum, 2005), it is reasonable to propose that any contractual, organisational, or anti-doping impediment to clinical psychedelic research should be carefully weighed up against the urgent need for new and effective mental health treatments.

\section{Current Psychedelic Use in Athletes}

While alcohol use and binge drinking is reported to be frequent, illicit drug use appears uncommon in elite athletes; though accurate prevalence rates are unclear and vary considerably by sport and other individual characteristics such as nationality and gender (McDuff et al., 2019). Cannabis appears the most commonly used illicit substance, with cocaine second despite its low prevalence (0.1-3.8\%) (McDuff et al., 2019). However, studies examining drug use in sport have generally neglected any in-depth exploration of psychedelics. Therefore, it will be of initial interest to understand: (1) what are the views of 
professionals in the sports industry on using psychedelics to treat mental ill-health; (2) what proportion of athletes currently, or have previously, used psychedelics, and with what motivations; and (3) what are the positive and/or negative psychological and performance correlates of extant psychedelic use in athletes.

\section{Randomized Controlled Trials: Efficacy, Safety, and Effects on Performance}

Once descriptive studies have helped inform our understanding of current views and practices around psychedelics in sport, high quality randomized controlled trials will be required to assess the benefits, safety, and specific requirements for elite athletes. Key outcomes of this work will be to determine not only the effects on mental health, but also on sport-specific performance, as discussed above. Many psychiatric treatments come with a range of negative side effects that can be particularly troublesome for athletes (Reardon, 2016). In the case of psychedelics, it is likely that this would be minimal, given their negligible physiological risks and toxicity, and because they are typically delivered as a short treatment program, rather than ongoing, as with other psychiatric medications. It will be important that clinicians who are involved in such work have significant and applied sportspecific therapeutic knowledge and understanding alongside clinical psychedelic training, in order for such work to be contextually aligned. Studies should examine whether psychedelics are beneficial for a range of mental health outcomes in athletes, both generally, and within the context of the previously described sport-specific stressors. One of a number of valuable initial trials within sport could recruit recently retired athletes who are experiencing psychosocial distress related to their retirement. This trial could investigate the effects of psilocybin-assisted psychotherapy on common (e.g., depression, anxiety) and sport-relevant (e.g., loss of identity and meaning, help-seeking behaviour) outcomes, while exploring a range of potential acute and enduring effects of sport-relevant performance. High levels of 
confidentiality would need to be exercised by research and clinical staff delivering psychedelic treatment to an athlete, in particular where the athlete is a public figure.

\section{Application in Practice}

Until we have sufficient safety and efficacy evidence, and a sufficient pool of adequately trained and experienced clinicians able to safely and effectively deliver psychedelic treatment, any application of psychedelics should be done within research trials only. If psychedelic medicines become regulated treatments and are subsequently approved by WADA, future delivery would likely occur in a specialist facility with close support from therapists trained in psychedelic-assisted psychotherapy. Psychedelic therapies are unique in the context of psychiatry in that a range of extra-pharmacological factors impact substantially on both safety and efficacy, including specific psychological preparation before, and integration following, dosing; a conducive mindset and physical environment during dosing; and the quality of the elicited altered state of consciousness. This treatment is also unique in the context of psychology, in its use of a psychotropic intervention. In the main, a collaborative care model between psychiatrists and psychologists will be required to deliver this treatment safely and effectively.

Moreover, sport psychologists have a wide ranging diversity in competencies and professional boundaries regarding mental health treatment and management, which often reflects where they were trained. While some sport psychologists have significant experience and interest in managing mental ill-health in athletes, others are more specifically focused on performance-based mental skills (Sly, Mellalieu, \& Wagstaff, 2020). Therefore, we would anticipate that the implementation of the described intervention in both research and practice will have varying levels of relevance and applicability to different sport psychologists. Given the requirement for specialist clinicians and settings for this treatment however, both sport psychologists and psychiatrists should only deliver this treatment following specialty 
training and in-practice supervision in psychedelic-assisted psychotherapy. In addition, the crucial role that a sport psychologist/psychiatrist may provide outside delivery of psychedelic treatments should not be overlooked. We suggest that they may be appropriately situated to be the primary point of coordination between the athlete, club, and psychedelic medicine facility. With athletes' consent, sport psychologists may also play a pivotal role in informing the specialist clinician on some of the relevant sport-specific factors and themes that may be addressed during therapy, based on their experience from a more established therapeutic relationship and understanding of elite sport stressors and processes. Sport psychiatrists may also be well aligned to assist in the ongoing management of athletes throughout the treatment phase, given their specialist training in medical management of athlete mental health (Currie and Johnston, 2016). Therefore, this treatment approach will, by its very nature, be multidisciplinary if implemented within sport.

Aside from the clinical delivery of treatment within these facilities, complex ethical decision-making regarding policy and protocol of athlete confidentiality will be needed. The community will need to decide whether the use of psychedelic-assisted psychotherapy will need to be declared to certain regulatory bodies, or may be able to be handled confidentially by the athletes' managing medical and psychology support team. Answers to these questions will only be possible once solid evidence regarding any potential negative or positive performance outcomes following use is obtained. Where coaching and support staff must be made aware of such involvement for contractual and organisational reasons, psychoeducation as to the potential benefits and risks of psychedelics is advised to reduce any chances of stigma and repercussions to the athlete. While discussing factors relevant to future service delivery, at present based on inadequate data, we do not promote the use of psychedelics for elite athletes outside of research trials.

\section{Conclusions}


This is the first paper to outline the therapeutic potential for psychedelic substances on mental health in athletes. Based on current early evidence, clinically-supported psychedelic treatments appear safe and highly efficacious across a growing number of mental health and addiction disorders. The field of psychedelic-assisted psychotherapy is in the midst of a global resurgence, with a rapidly growing number of high-quality clinical trials and the impending prospect of licencing some of these treatments. Should this occur, public and clinical interest is likely to be high, given the field is already attracting enormous amounts of attention and investment. In light of this upcoming and potentially impactful addition to the psychiatric arsenal, mental healthcare professionals who work with athletes should be aware of this growing field, and the sport-specific clinical potential and regulatory challenges that need to be navigated. In addition, clinical trials should be conducted to assess the safety and efficacy of this treatment approach in elite athletes. Key regulatory and performance-enhancing questions must be answered with respect to research and treatment with currently competing athletes; these specific challenges, however, would not apply to retired athletes experiencing mental health problems. The field of sports psychology and psychiatry would be well advised to take note of this promising research area, and to begin dialogue on how to best address the challenges and explore the opportunities of psychedelic medicine for elite athletes. 


\section{References}

Aday, J. S., Mitzkovitz, C. M., Bloesch, E. K., Davoli, C. C., \& Davis, A. K. (2020). Long-term effects of psychedelic drugs: A systematic review. Neuroscince and Biobehavioural Reviews, 113, 179-189. doi:10.1016/j.neubiorev.2020.03.017

Åkesdotter, C., Kenttä, G., Eloranta, S., \& Franck, J. (2020). The prevalence of mental health problems in elite athletes. Journal of Science and Medicine in Sport, 23(4), 329-335. doi:https://doi.org/10.1016/j.jsams.2019.10.022

Arnold, R., \& Fletcher, D. (2012). A Research Synthesis and Taxonomic Classification of the Organizational Stressors Encountered by Sport Performers. Journal oSport and Exercise Psychology, 34(3), 397-429. doi:10.1123/jsep.34.3.397

Baranoff, J., Hanrahan, S. J., \& Connor, J. P. (2015). The roles of acceptance and catastrophizing in rehabilitation following anterior cruciate ligament reconstruction. Journal of Science and Medicine in Sport, 18(3), 250-254. doi:10.1016/j.jsams.2014.04.002

Baum, A. L. (2005) Suicide in athletes: a review and commentary. Clinics in Sports Medicine, 24(4):853-69. doi: 10.1016/j.csm.2005.06.006.

Belser, A. B., Agin-Liebes, G., Swift, T. C., Terrana, S., Devenot, N., Friedman, H. L., .. Ross, S. (2017). Patient Experiences of Psilocybin-Assisted Psychotherapy: An Interpretative Phenomenological Analysis. Journal of Humanistic Psychology, 57(4), 354-388. doi:10.1177/0022167817706884

Bogenschutz, M. P., Forcehimes, A. A., Pommy, J. A., Wilcox, C. E., Barbosa, P. C., \& Strassman, R. J. (2015). Psilocybin-assisted treatment for alcohol dependence: a proof-of-concept study. Journal of Psychopharmacology, 29(3), 289-299. doi:10.1177/0269881114565144

Bonomo, Y., Norman, A., Biondo, S., Bruno, R., Daglish, M., Dawe, S., . . Castle, D. (2019). The Australian drug harms ranking study. Journal of Psychopharmacology, 33(7), 759-768. doi:10.1177/0269881119841569

Brewer, B. W., Cornelius, A. E., Stephan, Y., \& Van Raalte, J. (2010). Self-protective changes in athletic identity following anterior cruciate ligament reconstruction. Psychology of Sport and Exercise, 11(1), 1-5. doi:10.1016/j.psychsport.2009.09.005.

Brewer, B. W., \& Petitpas, A. J. (2017). Athletic identity foreclosure. Current Opinion in Psychology, 16, 118-122. doi:10.1016/j.copsyc.2017.05.004 
Carbonaro, T. M., Bradstreet, M. P., Barrett, F. S., MacLean, K. A., Jesse, R., Johnson, M. W., \& Griffiths, R. R. (2016). Survey study of challenging experiences after ingesting psilocybin mushrooms: Acute and enduring positive and negative consequences. Journal of Psychopharmacology, 30(12), 1268-1278. doi:10.1177/0269881116662634

Carhart-Harris, R., Leech, R., Hellyer, P., Shanahan, M., Feilding, A., Tagliazucchi, E., ... Nutt, D. (2014). The entropic brain: a theory of conscious states informed by neuroimaging research with psychedelic drugs. Frontiers in Human Neuroscience, 8(20). doi:10.3389/fnhum.2014.00020

Carhart-Harris, R. L. (2018). The entropic brain - revisited. Neuropharmacology, 142, 167-178. doi:10.1016/j.neuropharm.2018.03.010

Carhart-Harris, R. L., Bolstridge, M., Day, C. M. J., Rucker, J., Watts, R., Erritzoe, D. E., .. . Nutt, D. J. (2018). Psilocybin with psychological support for treatment-resistant depression: six-month follow-up. Psychopharmacology, 235(2), 399-408. doi:10.1007/s00213-017-4771-x

Carhart-Harris, R. L., Bolstridge, M., Rucker, J., Day, C. M. J., Erritzoe, D., Kaelen, M., ... Nutt, D. J. (2016). Psilocybin with psychological support for treatment-resistant depression: an open-label feasibility study. The Lancet Psychiatry, 3(7), 619-627. doi: 10.1016/S2215-0366(16)30065-7

Carhart-Harris, R. L., \& Goodwin, G. M. (2017). The Therapeutic Potential of Psychedelic Drugs: Past, Present, and Future. Neuropsychopharmacology, 42(11), 2105-2113. doi:10.1038/npp.2017.84

Carhart-Harris, R. L., Leech, R., Williams, T. M., Erritzoe, D., Abbasi, N., Bargiotas, T., .. . Nutt, D. J. (2012). Implications for psychedelic-assisted psychotherapy: functional magnetic resonance imaging study with psilocybin. British Journal of Psychiatry, 200(3), 238-244. doi:10.1192/bjp.bp.111.103309

Carhart-Harris, R. L., Roseman, L., Haijen, E., Erritzoe, D., Watts, R., Branchi, I., \& Kaelen, M. (2018). Psychedelics and the essential importance of context. Journal of Psychopharmacology, 32(7), 725-731. doi:10.1177/0269881118754710

Cavnar, C. (2014). The effects of ayahuasca ritual participation on gay and lesbian identity. J Psychoactive Drugs, 46(3), 252-260. doi:10.1080/02791072.2014.920117 
Chang, C., Putukian, M., Aerni, G., Diamond, A., Hong, G., Ingram, Y., ... Wolanin, A. (2020). Mental health issues and psychological factors in athletes: detection, management, effect on performance and prevention: American Medical Society for Sports Medicine Position Statement-Executive Summary. British Journal of Sports Medicine 54(4), 216-220. doi:10.1136/bjsports-2019-101583

Cohen, S. (1967). The beyond within: The LSD story: Atheneum New York.

Cohen, S. (1960). Lysergic acid diethylamide: side effects and complications. The Journal of Nervous and Mental Disease, 130(1), 30-40. doi:10.1097/00005053196001000-00005.

Currie, A., \& Johnston, A. (2016). Psychiatric disorders: The psychiatrist's contribution to sport. International Review of Psychiatry, 28(6), 587-594. doi:10.1080/09540261.2016.1197188

Doherty, S., Hannigan, B., \& Campbell, M. J. (2016). The Experience of Depression during the Careers of Elite Male Athletes. Frontiers in Psychology, 7(1069). doi:10.3389/fpsyg.2016.01069

Dolder, P. C., Schmid, Y., Müller, F., Borgwardt, S., \& Liechti, M. E. (2016). LSD Acutely Impairs Fear Recognition and Enhances Emotional Empathy and Sociality. Neuropsychopharmacology, 41(11), 2638-2646. doi:10.1038/npp.2016.82

Donohue, B., Gavrilova, Y., Galante, M., Gavrilova, E., Loughran, T., Scott, J., . . Allen, D. N. (2018). Controlled Evaluation of an Optimization Approach to Mental Health and Sport Performance. Journal of Clinical Sport Psychology, 12(2), 234-267. doi:10.1123/jcsp.2017-0054

Fadiman, J. (2011). The psychedelic explorer's guide: Safe, therapeutic, and sacred journeys: Simon and Schuster.

Garcia-Romeu, A., Davis, A. K., Erowid, F., Erowid, E., Griffiths, R. R., \& Johnson, M. W. (2019). Cessation and reduction in alcohol consumption and misuse after psychedelic use. Journal of Psychopharmacology, 33(9), 1088-1101. doi:10.1177/0269881119845793

Gasser, P., Holstein, D., Michel, Y., Doblin, R., Yazar-Klosinski, B., Passie, T., \& Brenneisen, R. (2014). Safety and efficacy of lysergic acid diethylamide-assisted psychotherapy for anxiety associated with life-threatening diseases. The Journal of Nervous and Mental Disease, 202(7), 513-520. doi:10.1097/nmd.0000000000000113 
Gouttebarge, V., Castaldelli-Maia, J. M., Gorczynski, P., Hainline, B., Hitchcock, M. E., Kerkhoffs, G. M., ... Reardon, C. L. (2019). Occurrence of mental health symptoms and disorders in current and former elite athletes: a systematic review and metaanalysis. British Journal of Sports Medicine, 53(11), 700-706. doi:10.1136/bjsports-2019-100671

Griffiths, R. R., Johnson, M. W., Carducci, M. A., Umbricht, A., Richards, W. A., Richards, B. D., ... Klinedinst, M. A. (2016). Psilocybin produces substantial and sustained decreases in depression and anxiety in patients with life-threatening cancer: A randomized double-blind trial. Journal of Psychopharmacology, 30(12), 11811197. doi:10.1177/0269881116675513

Griffiths, R. R., Johnson, M. W., Richards, W. A., Richards, B. D., Jesse, R., MacLean, K. A., . . . Klinedinst, M. A. (2018). Psilocybin-occasioned mystical-type experience in combination with meditation and other spiritual practices produces enduring positive changes in psychological functioning and in trait measures of prosocial attitudes and behaviors. Journal of Psychopharmacology, 32(1), 49-69. doi:10.1177/0269881117731279

Griffiths, R. R., Richards, W. A., McCann, U., \& Jesse, R. (2006). Psilocybin can occasion mystical-type experiences having substantial and sustained personal meaning and spiritual significance. Psychopharmacology, 187(3), 268-283. doi:10.1007/s00213-006-0457-5

Grinspoon, L., \& Bakalar, J. B. (1979). Psychedelic drugs reconsidered: Basic Books New York.

Grinspoon, L., \& Bakalar, J. B. (1981). The psychedelic drug therapies. Current Psychiatric Therapies, 20, 275-283.

Grob, C. S., Danforth, A. L., Chopra, G. S., Hagerty, M., McKay, C. R., Halberstadt, A. L., \& Greer, G. R. (2011). Pilot study of psilocybin treatment for anxiety in patients with advanced-stage cancer. Archives of General Psychiatry, 68(1), 71-78. doi:10.1001/archgenpsychiatry.2010.116

Gulliver, A., Griffiths, K. M., \& Christensen, H. (2012). Barriers and facilitators to mental health help-seeking for young elite athletes: a qualitative study. BMC Psychiatry, 12(1), 157. doi:10.1186/1471-244x-12-157

Hofmann, A., \& Ott, J. (1980). LSD, my problem child (Vol. 5): McGraw-Hill New York. 
Johansen, P. O., \& Krebs, T. S. (2015). Psychedelics not linked to mental health problems or suicidal behavior: a population study. Journal of Psychopharmacology, 29(3), 270-279. doi:10.1177/0269881114568039

Johnson, M., Richards, W., \& Griffiths, R. (2008). Human hallucinogen research: guidelines for safety. Journal of Psychopharmacology, 22(6), 603-620. doi:10.1177/0269881108093587

Johnson, M. W., Garcia-Romeu, A., Cosimano, M. P., \& Griffiths, R. R. (2014). Pilot study of the 5-HT2AR agonist psilocybin in the treatment of tobacco addiction. Journal of Psychopharmacology, 28(11), 983-992. doi:10.1177/0269881114548296

Johnson, M. W., Griffiths, R. R., Hendricks, P. S., \& Henningfield, J. E. (2018). The abuse potential of medical psilocybin according to the 8 factors of the Controlled Substances Act. Neuropharmacology, 142, 143-166. doi:10.1016/j.neuropharm.2018.05.012.

Jungaberle, H., Thal, S., Zeuch, A., Rougemont-Bücking, A., von Heyden, M., Aicher, H., \& Scheidegger, M. (2018). Positive psychology in the investigation of psychedelics and entactogens: A critical review. Neuropharmacology, 142, 179-199. doi: 10.1016/j.neuropharm.2018.06.034

Keyes, C. L. (2002). The mental health continuum: From languishing to flourishing in life. Journal of Health and Social Behavior, 43(2), 207-222.

Krebs, T. S., \& Johansen, P.-Ø. (2013). Psychedelics and Mental Health: A Population Study. PLoS One, 8(8), e63972. doi:10.1371/journal.pone.0063972

Lerner, A. G., Gelkopf, M., Skladman, I., Oyffe, I., Finkel, B., Sigal, M., \& Weizman, A. (2002). Flashback and Hallucinogen Persisting Perception Disorder: clinical aspects and pharmacological treatment approach. Israel Journal of Psychiatry and Related Sciences, 39(2), 92-99.

Luoma, J. B., Sabucedo, P., Eriksson, J., Gates, N., \& Pilecki, B. C. (2019). Toward a contextual psychedelic-assisted therapy: Perspectives from Acceptance and Commitment Therapy and contextual behavioral science. Journal of Contextual Behavioral Science, 14, 136-145. doi: 10.1016/j.jcbs.2019.10.003

MacLean, K. A., Johnson, M. W., \& Griffiths, R. R. (2011). Mystical experiences occasioned by the hallucinogen psilocybin lead to increases in the personality domain of openness. Journal of Psychopharmacology, 25(11), 1453-1461. doi:10.1177/0269881111420188 
Mannes, Z. L., Waxenberg, L. B., Cottler, L. B., Perlstein, W. M., Burrell, L. E., Ferguson, E. G., ... Ennis, N. (2018). Prevalence and correlates of psychological distress among retired elite athletes: A systematic review. International Review of Sport and Exercise Psychology, 12(1), 1-30. doi:10.1080/1750984X.2018.1469162

McDuff, D., Stull, T., Castaldelli-Maia, J. M., Hitchcock, M. E., Hainline, B., \& Reardon, C. L. (2019). Recreational and ergogenic substance use and substance use disorders in elite athletes: a narrative review. British Journal of Sports Medicine, 53(12), 754760. doi:10.1136/bjsports-2019-100669

Meikle, S. E., Liknaitzky, P., Rossell, S. L., Ross, M., Strauss, N., Thomas, N., . . Castle, D. J. (2019). Psilocybin-assisted therapy for depression: How do we advance the field? Australian \& New Zealand Journal of Psychiatry, 54(3), 225-231. doi:10.1177/0004867419888575

Moreland, J. J., Coxe, K. A., \& Yang, J. (2018). Collegiate athletes' mental health services utilization: A systematic review of conceptualizations, operationalizations, facilitators, and barriers. Journal of Sport and Health Science, 7(1), 58-69. doi: 10.1016/j.jshs.2017.04.009

Moreno, F. A., Wiegand, C. B., Taitano, E. K., \& Delgado, P. L. (2006). Safety, tolerability, and efficacy of psilocybin in 9 patients with obsessive-compulsive disorder. Journal of Clinical Psychiatry, 67(11), 1735-1740. doi:10.4088/jcp.v67n1110

Nichols, D. E. (2016). Psychedelics. Pharmacological Reviews, 68(2), 264-355. doi:10.1124/pr.115.011478

Nutt, D., Erritzoe, D., \& Carhart-Harris, R. (2020). Psychedelic Psychiatry's Brave New World. Cell, 181(1), 24-28. doi:10.1016/j.cell.2020.03.020

Nutt, D. J., King, L. A., \& Phillips, L. D. (2010). Drug harms in the UK: a multicriteria decision analysis. The Lancet, 376(9752), 1558-1565. doi:10.1016/S01406736(10)61462-6

Oroc, J. (2011). Psychedelics and Extreme Sports. Multidisciplinary Association for Psychedelic Studies Bulletin, 21(1), 25-29.

Osmond, H. (1957). A review of the clinical effects of psychotomimetic agents. Annals of the New York Academy of Sciences, 66(3), 418-434. doi:10.1111/j.17496632.1957.tb40738.x 
Park, S., Lavallee, D., \& Tod, D. (2013). Athletes' career transition out of sport: A systematic review. International Review of Sport and Exercise Psychology, 6(1), 22-53. Doi:10.1080/1750984X.2012.687053

Polito, V., \& Stevenson, R. J. (2019). A systematic study of microdosing psychedelics. PLoS One, 14(2), e0211023. doi:10.1371/journal.pone.0211023

Poucher, Z. A., Tamminen, K. A., Kerr, G., \& Cairney, J. (2019). A Commentary on Mental Health Research in Elite Sport. Journal of Applied Sport Psychology, 1-23. doi:10.1080/10413200.2019.1668496

Preller, K. H., Herdener, M., Pokorny, T., Planzer, A., Kraehenmann, R., Stämpfli, P., .. . Vollenweider, F. X. (2017). The Fabric of Meaning and Subjective Effects in LSDInduced States Depend on Serotonin 2A Receptor Activation. Current Biology, 27(3), 451-457. doi:10.1016/j.cub.2016.12.030

Preller, K. H., Pokorny, T., Krähenmann, R., Dziobek, I., Stämpfli, P., \& Vollenweider, F. X. (2015). The Effect of 5-HT2A/1a Agonist Treatment On Social Cognition, Empathy, and Social Decision-making. European Psychiatry, 30, 22. doi: 10.1016/S0924-9338(15)30017-1

Purcell, R., Gwyther, K., \& Rice, S. M. (2019). Mental Health In Elite Athletes: Increased Awareness Requires An Early Intervention Framework to Respond to Athlete Needs. Sports Medicine Open, 5(1), 46. doi:10.1186/s40798-019-0220-1

Purcell, R., Rice, S., Butterworth, M., \& Clements, M. (2020). Rates and Correlates of Mental Health Symptoms in Currently Competing Elite Athletes from the Australian National High-Performance Sports System. Sports Medicine, 50(9), 1683-1694, doi:10.1007/s40279-020-01266-z

Puspanathan, P. (2017). Psychedelic research in Australia: Breaking through the stigma. Austrailian and New Zealand Journal of Psychiatry, 51(9), 940-941. doi:10.1177/0004867417701580

Reardon, C. L. (2016). The sports psychiatrist and psychiatric medication. International Review of Psychiatry, 28(6), 606-613. doi:10.1080/09540261.2016.1190691

Reardon, C. L., Hainline, B., Aron, C. M., Baron, D., Baum, A. L., Bindra, A., .. Engebretsen, L. (2019). Mental health in elite athletes: International Olympic Committee consensus statement (2019). British Journal of Sports Medicine, 53(11), 667-699. doi:10.1136/bjsports-2019-100715 
Reiff, C. M., Richman, E. E., Nemeroff, C. B., Carpenter, L. L., Widge, A. S., Rodriguez, C. I., . . . The Work Group on Biomarkers and Novel Treatments, a Division of the American Psychiatric Association Council of Research. (2020). Psychedelics and Psychedelic-Assisted Psychotherapy. American Journal of Psychiatry, 177(5), 399410. doi:10.1176/appi.ajp.2019.19010035

Rice, S. M., Purcell, R., De Silva, S., Mawren, D., McGorry, P. D., \& Parker, A. G. (2016). The Mental Health of Elite Athletes: A Narrative Systematic Review. Sports Medicine, 46(9), 1333-1353. doi:10.1007/s40279-016-0492-2

Rice, S. M., Purcell, R., \& McGorry, P. D. (2018). Adolescent and Young Adult Male Mental Health: Transforming System Failures Into Proactive Models of Engagement. Journal of Adolescent Health, 62(3S), S9-S17. doi:10.1016/j.jadohealth.2017.07.024

Ronkainen, N. J., Kavoura, A., \& Ryba, T. V. (2016). A meta-study of athletic identity research in sport psychology: Current status and future directions. International Review of Sport and Exercise Psychology, 9(1), 45-64. doi:10.1080/1750984X.2015.1096414

Rosenbaum, D., Boyle, A. B., Rosenblum, A. M., Ziai, S., Chasen, M. R., \& Med, M. P. (2019). Psychedelics for psychological and existential distress in palliative and cancer care. Current Oncology, 26(4), 225-226. doi:10.3747/co.26.5009

Ross, S. (2018). Therapeutic use of classic psychedelics to treat cancer-related psychiatric distress. International Review of Psychiatry, 30(4), 317-330. doi:10.1080/09540261.2018.1482261

Ross, S., Bossis, A., Guss, J., Agin-Liebes, G., Malone, T., Cohen, B., .. Schmidt, B. L. (2016). Rapid and sustained symptom reduction following psilocybin treatment for anxiety and depression in patients with life-threatening cancer: a randomized controlled trial. Journal of Psychopharmacology, 30(12), 1165-1180. doi:10.1177/0269881116675512

Rucker, J. J. H., Iliff, J., \& Nutt, D. J. (2018). Psychiatry \& the psychedelic drugs. Past, present \& future. Neuropharmacology, 142, 200-218. doi:10.1016/j.neuropharm.2017.12.040

Sampedro, F., de la Fuente Revenga, M., Valle, M., Roberto, N., Domínguez-Clavé, E., Elices, M., ... Riba, J. (2017). Assessing the Psychedelic "After-Glow" in Ayahuasca Users: Post-Acute Neurometabolic and Functional Connectivity 
Changes Are Associated with Enhanced Mindfulness Capacities. International Journal of Neuropsychopharmacology, 20(9), 698-711. doi:10.1093/ijnp/pyx036

Schmid, Y., Enzler, F., Gasser, P., Grouzmann, E., Preller, K. H., Vollenweider, F. X., . . Liechti, M. E. (2015). Acute Effects of Lysergic Acid Diethylamide in Healthy Subjects. Biological Psychiatry, 78(8), 544-553. doi:10.1016/j.biopsych.2014.11.015

Schultes, R. E. (1969). Hallucinogens of plant origin. Science, 163(3864), 245-254. doi:10.1126/science.163.3864.245

Sellers, E.M. and Leiderman, D.B. (2018), Psychedelic Drugs as Therapeutics: No Illusions About the Challenges. Clinical Pharmacology \& Therapeutics, 103: 561564. doi:10.1002/cpt.776

Sessa, B. (2015). Turn on and tune in to evidence-based psychedelic research. The Lancet Psychiatry, 2(1), 10-12. doi:10.1016/S2215-0366(14)00120-5

Shanon, B. (2010). The epistemics of ayahuasca visions. Phenomenology and the Cognitive Sciences, 9(2), 263-280. doi:10.1007/s11097-010-9161-3

Sly, D., Mellalieu, S. D., \& Wagstaff, C. R. (2020). "It's psychology Jim, but not as we know it!": The changing face of applied sport psychology. Sport, Exercise, and Performance Psychology, 9(1), 87-101. Doi:10.1037/spy0000163

Stillman, M. A., Glick, I. D., McDuff, D., Reardon, C. L., Hitchcock, M. E., Fitch, V. M., \& Hainline, B. (2019). Psychotherapy for mental health symptoms and disorders in elite athletes: a narrative review. British Journal of Sports Medicine, 53(12), 767771. doi:10.1136/bjsports-2019-100654

Strauss, N., Bright, S. J., \& Williams, M. L. (2016). Australia should be initiating a psychedelic research program: What are the barriers? Australian \& New Zealand Journal of Psychiatry, 50(11), 1036-1037. doi:10.1177/0004867416670520

Uphill, M., Sly, D., \& Swain, J. (2016). From Mental Health to Mental Wealth in Athletes: Looking Back and Moving Forward. Frontiers in Psychology, 7(935). doi:10.3389/fpsyg.2016.00935

van Amsterdam, J., Nutt, D., Phillips, L., \& van den Brink, W. (2015). European rating of drug harms. Journal of Psychopharmacology, 29(6), 655-660. doi:10.1177/0269881115581980 
Vollenweider, F. X., \& Preller, K. H. (2020). Psychedelic drugs: neurobiology and potential for treatment of psychiatric disorders. Nature Reviews Neuroscience, 21(11), 611-624. doi:10.1038/s41583-020-0367-2

Wachsmuth, S., Jowett, S., \& Harwood, C. G. (2017). Conflict among athletes and their coaches: what is the theory and research so far? International Review of Sport and Exercise Psychology, 10(1), 84-107. doi:10.1080/1750984X.2016.1184698

WADA. (2020). World Anti-Doping Code. Retrieved on 02/10/2020 from https://www.wada-ama.org/en/resources/science-medicine/prohibited-listdocuments

Walton, C. C., Baranoff, J., Gilbert, P., \& Kirby, J. (2020). Self-compassion, social rank, and psychological distress in athletes of varying competitive levels. Psychology of Sport and Exercise, 50, 101733. doi:10.1016/j.psychsport.2020.101733

Watson, J. C. (2005). College Student-Athletes' Attitudes Toward Help-Seeking Behavior and Expectations of Counseling Services. Journal of College Student Development, 46(4), 442-449. doi:10.1353/csd.2005.0044

Watts, R., Day, C., Krzanowski, J., Nutt, D., \& Carhart-Harris, R. (2017). Patients' Accounts of Increased “Connectedness" and "Acceptance” After Psilocybin for TreatmentResistant Depression. Journal of Humanistic Psychology, 57(5), 520-564. doi:10.1177/0022167817709585 


\section{University Library}

\section{- M M I N E R VA A gateway to Melbourne's research publications}

Minerva Access is the Institutional Repository of The University of Melbourne

Author/s:

Walton, CC;Liknaitzky, P

Title:

Advancing elite athlete mental health treatment with psychedelic-assisted psychotherapy

Date:

2020-11-13

Citation:

Walton, C. C. \& Liknaitzky, P. (2020). Advancing elite athlete mental health treatment with psychedelic-assisted psychotherapy. JOURNAL OF APPLIED SPORT PSYCHOLOGY, 34 (3), pp.605-623. https://doi.org/10.1080/10413200.2020.1848941.

Persistent Link:

http://hdl.handle.net/11343/251800 